\title{
QCD Thermodynamics and Magnetization in Nonzero Magnetic Field
}

\author{
Abdel Nasser Tawfik, ${ }^{1,2}$ Abdel Magied Diab, ${ }^{1,2}$ Nada Ezzelarab, ${ }^{1,2}$ and Asmaa G. Shalaby ${ }^{2,3}$ \\ ${ }^{1}$ Egyptian Center for Theoretical Physics (ECTP), Modern University for Technology and Information (MTI), Cairo 11571, Egypt \\ ${ }^{2}$ World Laboratory for Cosmology And Particle Physics (WLCAPP), Cairo 11571, Egypt \\ ${ }^{3}$ Physics Department, Benha University, Benha 13815, Egypt
}

Correspondence should be addressed to Abdel Nasser Tawfik; a.tawfik@eng.mti.edu.eg

Received 7 October 2015; Accepted 26 January 2016

Academic Editor: Juan José Sanz-Cillero

Copyright (C) 2016 Abdel Nasser Tawfik et al. This is an open access article distributed under the Creative Commons Attribution License, which permits unrestricted use, distribution, and reproduction in any medium, provided the original work is properly cited. The publication of this article was funded by SCOAP ${ }^{3}$.

\begin{abstract}
In nonzero magnetic field, the magnetic properties and thermodynamics of the quantum-chromodynamic (QCD) matter are studied in the hadron resonance gas and the Polyakov linear-sigma models and compared with recent lattice calculations. Both models are fairly suited to describe the degrees of freedom in the hadronic phase. The partonic ones are only accessible by the second model. It is found that the QCD matter has paramagnetic properties, which monotonically depend on the temperature and are not affected by the hadron-quark phase transition. Furthermore, raising the magnetic field strength increases the thermodynamic quantities, especially in the hadronic phase, but reduces the critical temperature, that is, inverse magnetic catalysis.
\end{abstract}

\section{Introduction}

In peripheral heavy-ion collisions, a strong and very localized magnetic field is likely generated. The opposite relativistic motion of the spectator's positive charges and the imbalance in the momentum of the participants together contribute to generating such short-lived and huge magnetic field $(B$ $10^{19}$ Gauss), which apparently should come up with significant effects on the quantum-chromodynamic (QCD) matter. These effects can be coupled to experimental observables; for instance, in the STAR experiment at the relativistic heavy-ion collider (RHIC), $|q| B \sim m_{\pi}^{2}[1,2]$, and in ALICE experiment at the large hadron collider (LHC), $|q| B \sim 10-15 m_{\pi}^{2}[1,2]$, where $|q|$ is the net electric charge and $m_{\pi}$ is the pion mass [3]. Only in heavy-ion collisions do the self-generating magnetic fields play an essential role. The early universe and magnetors (special types of neutron stars), for instance, should extremely be affected by such fields, which are conjectured to influence even the acceleration of various cosmic rays and the creation of stars [4]. They can mediate important processes affecting the dynamics, the distribution, and even the composition of the galactic plasmas, for instance [4].
The influences of magnetic fields on the hadronic matter and on the phase-space structure of quark-gluon plasma (QGP) are included in various models, such as hadron resonance gas (HRG) $[5,6]$, and estimated in lattice QCD simulations [7-12]. The Polyakov-Nambu-Jona-Lasinio (PNJL) and NJL models are examples on QCD-like models in which such magnetic effects were estimated [13-16]. Coupling Polyakov loops to the linear-sigma model (PLSM) introduces color charge interactions to the pure gauge field. PLSM reveals interesting features about the response of QCD matter to finite magnetic field $[17,18]$. Recently, electric conductivity in thermal medium and the phase structure of the strongly interacting matter in presence of magnetic field have been reported [19-22].

In the present work, we plan to utilize the HRG and PLSM approaches in finite magnetic field in order to study the QCD equation-of-state (EoS). Furthermore, it intends to estimate different thermodynamic observables including pressure, entropy, energy densities, and magnetization by using the modified energy-momentum dispersion relations which arise from finite magnetic field. Also, we verify that the thermal QCD medium is paramagnetic, especially at 
the critical temperature. Our calculations are confronted to recent lattice QCD simulations [12]. A quite good agreement is observed.

The present paper is organized as follows. We summarize the fundamentals of Landau quantizations in Section 2.1. The HRG model in nonzero magnetic field is discussed in Section 2.2. PLSM is briefly introduced in Section 2.3. The obtained results are confronted to recent lattice QCD in Section 3. The conclusions are outlined in Section 4.

\section{Formalism}

2.1. General Remarks on Landau Quantization. In nonzero magnetic field, the eigenenergy can be given as

$$
E=\left(n+\frac{1}{2} \hbar \omega_{H}\right)+\frac{p_{z}^{2}}{2 m}-\mu \frac{S_{z}}{s} H,
$$

where the angular frequency $\omega_{H}=|e| H / m c$ and discrete values with integer quantum number $n=0,1,2, \ldots$ called Landau levels are assigned to the first term [23, 24]. This describes the motion in a plane perpendicular to the magnetic field, which is directed towards the $z$-axis. Accordingly, the dispersion relation should be modified [23-25]:

$$
E_{\ell S_{z}}=\left[p_{z}^{2}+m^{2}+2|q|\left(n-S_{z}+\frac{1}{2}\right) B\right]^{1 / 2},
$$

where $S_{z}$ is the component of the spin in the $z$-direction, $n$ being the index to label the Landau levels, and $|q|>0$ is the electric charge of the $i$ th hadron. In PLSM, the dispersion relation also becomes modified:

$$
E_{B, f}(B)=\left[p_{z}^{2}+m_{f}^{2}+\left|q_{f}\right|(2 n+1-\sigma) B\right]^{1 / 2},
$$

where the quantization number $(n)$ known as the Landau quantum number, $\sigma$, is related to the spin quantum number, $\sigma= \pm S_{z} / 2$ and $q_{f}\left(m_{f}\right)$ being quark electric charge (mass). The quark masses are directly coupled to the $\sigma$-fields through Yukawa coupling $g$ as

$$
\begin{aligned}
& m_{l}=g \frac{\sigma_{l}}{2}, \\
& m_{s}=g \frac{\sigma_{s}}{\sqrt{2}},
\end{aligned}
$$

where the subscript $l$ refers to degenerate light up and down quarks.
It is worthwhile to notice that $2 n+1-\sigma$ can be replaced by sum over the Landau levels $0 \leq v \leq v_{\max _{f}}$. The lower value refers to the lowest Landau level (LLL), while the higher one stands for the maximum Landau level (MLL), $v_{\max }$, which contributes to the maximum quantization number $\left(\nu_{\max _{f}} \rightarrow\right.$ $\infty)$. For the sake for completeness, we mention that $2-\delta_{0 v}$ represents degenerate Landau levels.

2.2. Hadron Resonance Gas Model in Nonzero Magnetic Field. Treating hadron resonances as a collision-free gas [26-30] allows the estimation of thermodynamic partition function. For a recent review, the readers can consult [31]. Even for an interacting system, such ideal gas, which is composed of hadron resonances with masses $\leq 2 \mathrm{GeV}[29,32]$, where the inclusion of heavy resonances effectively introduces interactions and correlations to the system, can be described by grand canonical partition function that gives a quite satisfactory description for the particle production in heavyion collisions and the lattice QCD thermodynamics [26$28,33,34]$

$$
\begin{aligned}
& \ln Z(T, V, \mu) \\
& \quad=\sum_{i} \pm \frac{V d_{i}}{2 \pi^{2}} \int_{0}^{\infty} p^{2} \ln \left[1 \pm \exp \left[\frac{\left(\mu_{i}-E_{i}\right)}{T}\right]\right] d p
\end{aligned}
$$

where \pm stands for fermions and bosons, respectively, and $V$ is the volume and $d_{i}$ is the degeneracy for the $i$ th hadron. As discussed earlier, the dispersion relation $\left(E_{i}\right)$ for charged hadrons in nonzero magnetic field is a subject of modification

(2) while, for neutral hadrons, $E_{i}=\sqrt{p^{2}+m_{i}^{2}}$.

In finite magnetic field, the phase-space integral in (5) is to be expressed as a one-dimensional integral (dimension reduction). The partition function for the $i$ th particle in presence of magnetic field can be written as

$$
\begin{aligned}
& \ln Z(T, V, \mu) \\
& = \pm \frac{V d_{i}}{2 \pi^{2}}\left|q_{i}\right| e B_{z} \sum_{n} \sum_{S_{z}} \int_{0}^{\infty} \ln \left[1 \pm \exp \left(\frac{\mu_{i}-E_{i}}{T}\right)\right] d p_{z}
\end{aligned}
$$

where $p_{z}$ is the component of the particle momentum along the direction of the magnetic field. The spin in the $z$-direction $\left(S_{z}\right)$ is running as $-S_{z},-S_{z}+1, \ldots,+S_{z}$, where $S_{z}$ is the resonance spin in the $z$-direction. For the $i$ th particle, the pressure, energy density, and entropy, respectively, read

$$
\begin{aligned}
& P_{i}= \pm \frac{d_{i} T}{2 \pi^{2}}\left|q_{i}\right| e B \sum_{\mathcal{K}} \sum_{S_{z}} \int_{0}^{\infty} \ln \left[1 \pm \exp \left(\frac{\mu_{i}-E_{i}}{T}\right)\right] d p_{z}, \\
& \varepsilon_{i}= \pm \frac{d_{i} T}{2 \pi^{2}}\left|q_{i}\right| e B \sum_{\mathcal{K}} \sum_{S_{z}} \int_{0}^{\infty} \frac{E_{i}}{\exp \left[\left(E_{i}-\mu_{i}\right) / T\right] \pm 1} d p_{z}, \\
& s_{i}= \pm \frac{d_{i}}{2 \pi^{2}}\left|q_{i}\right| e B \sum_{\kappa} \sum_{S_{z}} \int_{0}^{\infty}\left\{\ln \left[1 \pm \exp \left(\frac{\mu_{i}-E_{i}}{T}\right)\right] \pm \frac{\left(E_{i}-\mu_{i}\right) / T}{\exp \left[\left(E_{i}-\mu_{i}\right) / T\right] \pm 1}\right\} d p_{z} .
\end{aligned}
$$


The magnetization (20) is a vector field indicating the creation of magnetic dipole moments resulting from the response of the material to nonzero magnetic field.

The dependence of hadron masses on nonzero magnetic field is still questionable [35]. In LHC, the magnetic field of noncentral heavy-ion collisions can reach up to $10-15 m_{\pi}^{2}$. This value is almost identical to $\Lambda_{\mathrm{QCD}}[1,36]$. At high momentum transfer, that is, asymptotic freedom, the QCD strength becomes very small at short distances, and the leading order running strong coupling reads [37]

$$
\frac{1}{\alpha_{s}} \simeq \beta_{0} \ln \left[\frac{|e B|}{\Lambda_{\mathrm{QCD}}^{2}}\right],
$$

where $\beta_{0}=\left(11 N_{c}-2 N_{f}\right) / 12 \pi$ and the QCD phase transition might take place at $\Lambda_{\mathrm{QCD}} \sim 0.2 \mathrm{GeV}$ or $B \sim 0.2 \mathrm{GeV}^{2}$. In this respect, the magnetic field can be categorized into

(i) $|e B| \gg \Lambda_{\mathrm{QCD}}^{2}$, strong magnetic field,

(ii) $|e B| \ll \Lambda_{\mathrm{QCD}}^{2}$, weak magnetic field.

On the other hand, in a finite magnetic field, the lattice QCD calculations refer to mass hierarchy [36]. It has been found that $m_{\rho^{0}} \sim m_{\pi^{+}}>m_{\rho^{+}} \sim m_{\pi^{0}}$ at $e B \gg \Lambda_{\mathrm{QCD}}^{2}$ [35]. Thus, the strong magnetic field is the one at which $\Lambda_{\mathrm{QCD}}^{2} \ll$ $|e B| \lesssim(10 \mathrm{TeV})^{2}$. This means that the value of $\Lambda_{\mathrm{QCD}}^{2}$ is small to contribute to the dynamical quark masses or the hadron constituents; that is, QCD represents an intermediate regime [36].

2.3. Polyakov Linear-Sigma Model in Nonzero Magnetic Field. In $\mathrm{SU}(3)_{L} \times \mathrm{SU}(3)_{R}$ symmetries, the Lagrangian of LSM for $N_{f}=3$ flavors $\left(u-, d\right.$-, and $s$-quarks) with $N_{c}=3$ color degrees of freedom is given as

$$
\mathscr{L}=\mathscr{L}_{\text {chiral }}-\mathscr{U}\left(\phi, \phi^{*}, T\right),
$$

where the chiral Lagrangian consists of two parts: fermionic and mesonic. Both couple to each other with a flavor-blind Yukawa coupling constant $g$ [38]:

$$
\begin{aligned}
\mathscr{L}_{q}= & \sum_{f} \overline{\psi_{f}}\left[i \gamma^{\mu} D_{\mu}-g T_{a}\left(\sigma_{a}+i \gamma_{5} \pi_{a}\right)\right] \psi_{f} \\
\mathscr{L}_{m}= & \operatorname{Tr}\left(\partial_{\mu} \Phi^{\dagger} \partial^{\mu} \Phi-m^{2} \Phi^{\dagger} \Phi\right)-\lambda_{1}\left[\operatorname{Tr}\left(\Phi^{\dagger} \Phi\right)\right]^{2} \\
& -\lambda_{2} \operatorname{Tr}\left(\Phi^{\dagger} \Phi\right)^{2}+c\left[\operatorname{Det}(\Phi)+\operatorname{Det}\left(\Phi^{\dagger}\right)\right] \\
& +\operatorname{Tr}\left[H\left(\Phi+\Phi^{\dagger}\right)\right]
\end{aligned}
$$

where $T_{a}=\lambda_{a} / 2$ with $a=0,1, \ldots, 8$ are the nine generators of the $U(3)$ symmetry group and $\lambda_{a}$ are the eight Gell-Mann matrices [39]. $\sigma_{a}$ are the scalar and $\pi_{a}$ are the pseudoscalar mesons,

$$
\Phi=T_{a} \phi_{a}=T_{a}\left(\sigma_{a}+i \pi_{a}\right)
$$

The spontaneous and explicit chiral symmetry breaking is given in more detail in [40-42].
The second term in (11), $\mathcal{U}\left(\phi, \phi^{*}, T\right)$, represents the Polyakov-loop effective potential [43], where the Polyakovloop fields, $\phi$ and $\phi^{*}$, are the order parameters for deconfinement phase transition $[44,45]$. In the present work, we implement a polynomial expansion in $\phi$ and $\phi^{*}$ [44-47]:

$$
\begin{aligned}
\frac{\mathcal{U}\left(\phi, \phi^{*}, T\right)}{T^{4}}= & -\frac{b_{2}(T)}{2}|\phi|^{2}-\frac{b_{3}}{6}\left(\phi^{3}+\phi^{* 3}\right) \\
& +\frac{b_{4}}{4}\left(\left|\phi^{2}\right|\right)^{2},
\end{aligned}
$$

where $b_{2}(T)=a_{0}+a_{1}\left(T_{0} / T\right)+a_{2}\left(T_{0} / T\right)^{2}+a_{3}\left(T_{0} / T\right)^{3}$ with $a_{0}=6.75, a_{1}=-1.95, a_{2}=2.625, a_{3}=-7.44, b_{3}=0.75$, and $b_{4}=7.5$ [44]. The deconfinement temperature $T_{0}$ is fixed at $270 \mathrm{MeV}$.

In the mean field approximation, the thermodynamic potential estimates the energy exchange between quarks and antiquarks at temperature $(T)$ and baryon chemical potential $\left(\mu_{f}\right)$, where $f$ runs over the quark flavors. The thermodynamic potential, $\Omega=-T \ln \mathscr{Z} / V$, reads

$$
\Omega=U\left(\sigma_{l}, \sigma_{s}\right)+\mathcal{U}\left(\phi, \phi^{*}, T\right)+\Omega_{\bar{q} q},
$$

where the first term represents the pure mesonic part

$$
\begin{aligned}
U\left(\sigma_{l}, \sigma_{s}\right)= & -h_{l} \sigma_{l}-h_{s} \sigma_{s}+\frac{m^{2}}{2}\left(\sigma_{l}^{2}+\sigma_{s}^{2}\right)-\frac{c}{2 \sqrt{2}} \sigma_{l}^{2} \sigma_{s} \\
& +\frac{\lambda_{1}}{2} \sigma_{l}^{2} \sigma_{s}^{2}+\frac{\left(2 \lambda_{1}+\lambda_{2}\right)}{8} \sigma_{l}^{4} \\
& +\frac{\left(\lambda_{1}+\lambda_{2}\right)}{4} \sigma_{s}^{4} .
\end{aligned}
$$

This potential can be constructed from $\sigma_{x}$ for light flavors $(u$ and $d$-quark) and $\sigma_{y}$ for $s$-quark.

In finite magnetic field (and finite $T$ and $\mu_{f}$ ) and by means of Landau quantization and magnetic catalysis, the quarkantiquark potential is given as

$$
\begin{aligned}
& \Omega_{\bar{q} q}\left(T, \mu_{f}, B\right)=-2 \sum_{f=l, s} \frac{\left|q_{f}\right| B T}{(2 \pi)^{2}} \sum_{\nu=0}^{\infty}\left(2-\delta_{0 \nu}\right) \int_{0}^{\infty} d p_{z} \\
& \cdot\left\{\operatorname { l n } \left[1+3\left(\phi+\phi^{*} e^{-\left(E_{B, f}-\mu_{f}\right) / T}\right) e^{-\left(E_{B, f}-\mu_{f}\right) / T}\right.\right. \\
& \left.+e^{-3\left(\left(E_{B, f}-\mu_{f}\right) / T\right)}\right]+\ln [1 \\
& +3\left(\phi^{*}+\phi e^{-\left(E_{B, f}+\mu_{f}\right) / T}\right) e^{-\left(E_{B, f}+\mu_{f}\right) / T} \\
& \left.\left.+e^{-3\left(\left(E_{B, f}+\mu_{f}\right) / T\right)}\right]\right\} .
\end{aligned}
$$

Finally, we should assume global minimization of the thermodynamic potential,

$$
\frac{\partial \Omega}{\partial \sigma_{l}}=\frac{\partial \Omega}{\partial \sigma_{s}}=\frac{\partial \Omega}{\partial \phi}=\left.\frac{\partial \Omega}{\partial \phi^{*}}\right|_{\min }=0,
$$

in order to fix the remaining parameters $\sigma_{l}=\overline{\sigma_{l}}, \sigma_{s}=\overline{\sigma_{s}}$, $\phi=\bar{\phi}$, and $\phi^{*}=\overline{\phi^{*}}$ and their dependencies on $T, \mu_{f}$, and $e B$ $[17,18]$. 


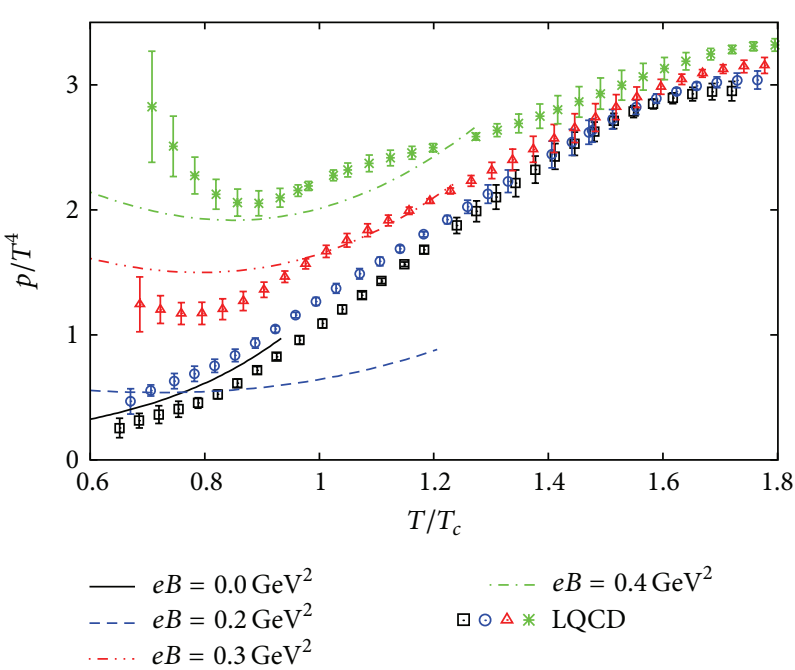

(a)

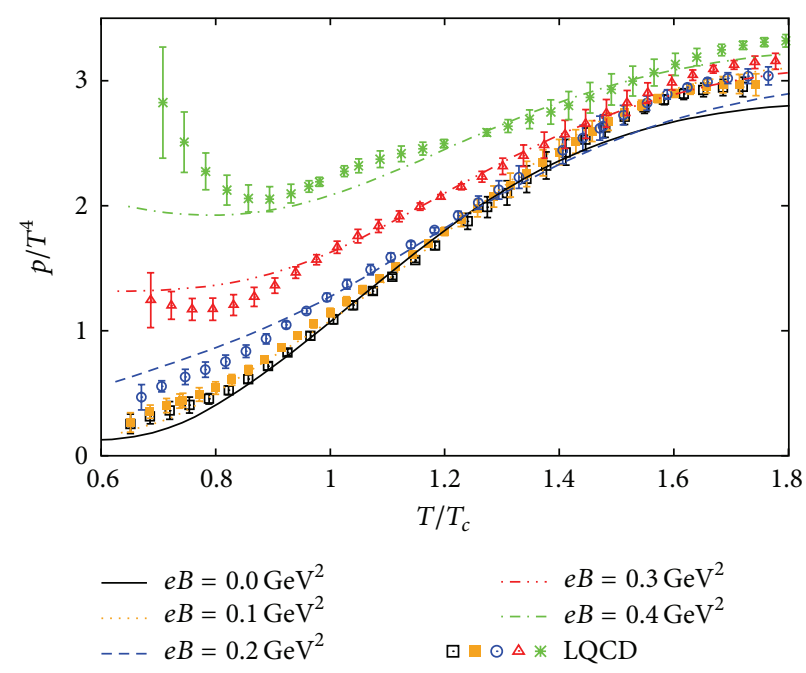

(b)

FIGURE 1: (a) The dependence of normalized pressure on temperature calculated from HRG model, (7) at different values of magnetic fields, $e B=0.0,0.1,0.2,0.3$, and $0.4 \mathrm{GeV}^{2}$ compared with recent lattice calculations [12] (symbols). (b) The same as in (a) but the calculations from PLSM (17).

\section{Results and Discussion}

Based on the remarkable success of the HRG model in reproducing lattice QCD thermodynamics, for instance, it is straightforward to perform similar analysis in nonzero magnetic field $[6,8]$. In peripheral heavy-ion collisions, the magnetic field can be as much as $e B=3.25 \mathrm{GeV}^{2}$ [48]. We also utilize PLSM in studying various QCD properties and phenomena [12]. The magnetization and different thermodynamic quantities are calculated from both models at $e B=0.0-0.4 \mathrm{GeV}^{2}$ and compared with recent lattice QCD calculations [12].

Figure 1 presents the normalized pressure $p / T^{4}$ as a function of temperature at $e B=0.0$ (solid), 0.2 (dashed), 0.3 (double-dot-dashed), and $0.4 \mathrm{GeV}^{2}$ (dot-dashed curve). The results are compared with recent lattice calculations [12] at $e B=0.0$ (open square), $e B=0.1$ (closed square), 0.2 (circle), 0.3 (triangle), and $0.4 \mathrm{GeV}^{2}$ (asterisk). (a) shows the calculations from the HRG model (curves), while the PLSM calculations are depicted in (b). It is apparent that the pressure increases with increasing magnetic field, especially at low temperatures. At high temperatures, $p / T^{4}$ is limited to the Stefan-Boltzmann limits, which apparently is not affected by the magnetic field.

In (a), we notice that the pressure calculated from HRG slightly deviates from the lattice calculations, although the temperature dependence is preserved. For HRG, $p(T, e B)$ is calculated from (7) without corrections due to lattice $\phi$ - and $B$-schemes [12]. Figure 1(b) shows the same as in (a) but for calculations from PLSM. Our results agree well with the lattice simulations [12] at a wide range of temperatures. The pressure is directly deduced from PLSM potential (17) in mean field approximation; that is, no corrections with respect to the magnetic flux $\left(\phi=e B \cdot L_{x} L_{y}\right)$ and/or the magnetic field $(B)$ have been done in lattice simulations, respectively.
It is obvious that the HRG calculations at low magnetic fields $\left(\lesssim 0.2 \mathrm{GeV}^{2}\right)$ disagree with the corresponding lattice simulations. They are almost temperature independent, which contradicts even the same calculations in vanishing magnetic field and becomes radical at large temperatures (near the critical temperature). It is worthwhile to recall that, in vanishing magnetic field, the partition function is given by integral over six-dimensional phase-space and the dispersion relations follow Lorentz invariance principle. But, in finite magnetic field, the integral dimensionality is drastically reduced and simultaneously accompanied by a considerable modification in the dispersion relation.

In finite magnetic field $B$, the velocity of a test particle with momentum $P$ can be deduced from the dispersion relations, $(2)$ and (3), respectively $\left(v=\partial \epsilon^{\text {total }} / \partial P\right)$ :

$$
\begin{aligned}
& v_{p}=c\left[\frac{c p}{c p+2|q|\left(\kappa+1 / 2-S_{z}\right) B}\right], \text { for HRG, } \\
& v_{p}=c\left[\frac{c p}{c p+2\left|q_{f}\right|(\kappa+1 / 2-\sigma / 2) B}\right], \text { for PLSM, }
\end{aligned}
$$

where $\sigma= \pm S / 2$. Then the causality is guaranteed for $v_{p}$ not exceeding the speed of light $c$, that is, as long as the $B$-term is finite positive, which should be estimated, quantitatively, as a function of temperature and magnetic field strength. This might give an explanation as to why HRG fails to reproduce lattice pressure at low magnetic field, while PLSM does not.

For a reliable comparison with the lattice calculations [12], an additional constant magnetic term should be added to the free energy, so that [5]

$$
\mathscr{M}=-\frac{1}{V} T \frac{\partial \ln \mathscr{Z}}{\partial(e B)} .
$$




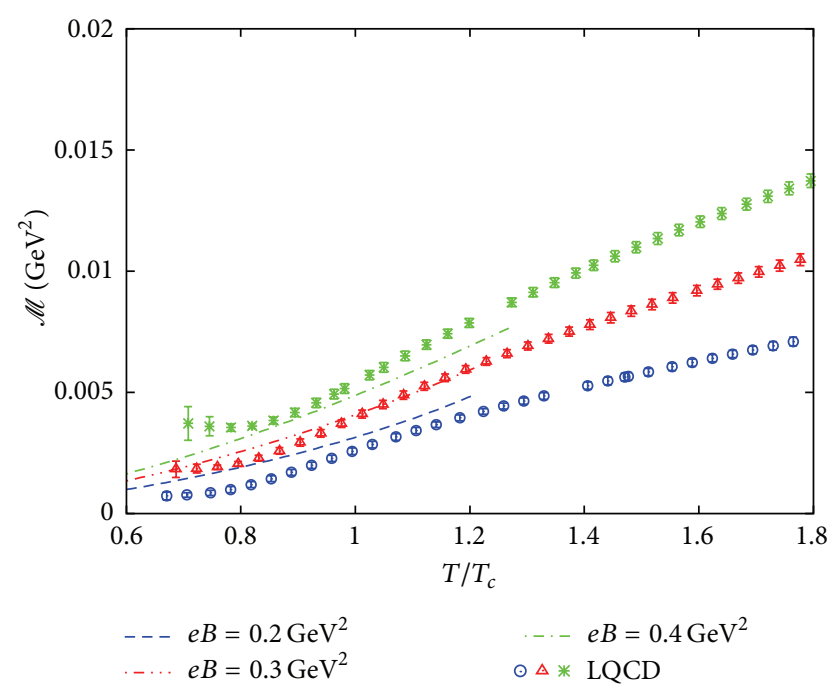

(a)

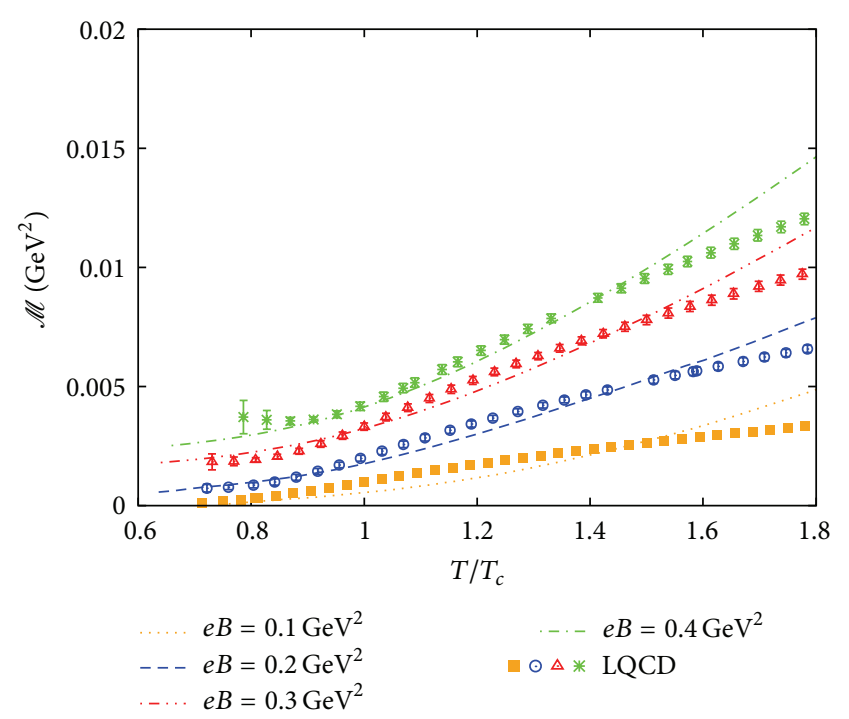

(b)

FIgURE 2: The magnetization $\mathscr{M}$ is given as a function of temperature at $e B=0.0-0.4 \mathrm{GeV}^{2}$ and compared with lattice results (symbols) [12]. The HRG results are depicted in (a), while those from PLSM are in (b).

Accordingly, one should distinguish between two setups where either magnetic field $B$ ( $B$-scheme) or magnetic flux $\Phi$ is kept constant during the expansion [12], where $L_{x}$ and $L_{y}$ are system extensions in the $x$ - and $y$-direction, respectively. The response of QCD matter (hadrons and partons) to nonzero magnetic field can be estimated from the magnetization.

In $B$-scheme, the pressure $p$ becomes isotropic but anisotropic in $\phi$-scheme. The compressing force in $\phi$-scheme is directed oppositely to the magnetic field. The longitudinal pressure $p_{z}$ does not depend on the scheme. The good agreement with lattice calculations, in Figure 1, especially at large magnetic fields, is obtained where the calculations are simply uncorrected or scheme-independent free-energies are assumed.

Sign of $\mathscr{M}$ defines the magnetic property of the system of interest. Paramagnetic property refers to attraction of QCD matter in external magnetic field. Diamagnetic QCD matter is slightly repelled by the magnetic field and does not retain its magnetic properties when the field is removed. Positive magnetization refers to paramagnetism, while negative $\mathscr{M}$ refers to diamagnetism. In Figure 2, the dependence of $\mathscr{M}$ on temperature is depicted at various $e B$. We notice that $\mathscr{M}$ has very small but positive values which indicate that the QCD matter has paramagnetic property and this behavior monotonically remains with increasing temperature. The phase transition at $T / T_{c}=1$ seems not affecting it. The magnetization from HRG fairly agrees with the lattice calculations, while PLSM excellently reproduces the lattice magnetization. The slight difference in both models should contribute to further thermodynamic quantities, Figure 3.

Figures 3(a) and 3(b) present the same as in Figure 1 but for total energy density

$$
\epsilon^{\text {total }}=\epsilon+e B \cdot \mathscr{M}
$$

where $\epsilon=-\beta \partial P / \partial \beta$ with $\beta=1 / T$ is the thermodynamic energy density. It is obvious that the agreement between HRG (a) and the lattice calculations is excellent, especially below $T_{c}$. Also, we observe that $T_{c}$ declines with increasing $e B$, that is, inverse magnetic catalysis in the hadronic matter, while $\epsilon^{\text {total }} / T^{4}$ remarkably increases with $e B$. In other words, we observe that increasing $e B$ leads to decreasing $T_{c}$. The values of $T_{c}$ can be deduced from the graphs. (b) shows the same but for calculations from PLSM. Here is fair agreement that is extended to a wider range of temperatures covering hadronic and partonic phases, simultaneously.

Figures 3(c) and 3(d) are the same as in Figure 1 but for entropy density $s=\left(\epsilon^{\text {total }}+p_{z}\right) / T$. In HRG, the increase in $s / T^{3}$ with increasing $e B$ is larger than in lattice calculations. The best agreement is found near $T_{c}$. In (d), entropy from PLSM is confronted to the lattice simulations. There is a nice agreement in both hadronic and partonic phases.

\section{Conclusions}

The introducing of finite magnetic field is accompanied with two types of modifications: first the phase-space should be changed due to inverse magnetic catalysis and second the dispersion relation as well as the distribution function should be modified. In mean field approximation, both Polyakov linearsigma model (PLSM) and hadron resonance gas (HRG) model are considered to study QCD thermodynamics in vanishing and finite magnetic field. The results from both models are confronted to recent lattice QCD calculations. In doing this, the temperature dependence of the magnetization should be determined. It seems that both models are fairly suited in describing the degrees of freedom in the hadronic phase, in which only PLSM contains the partonic degrees of freedom. 


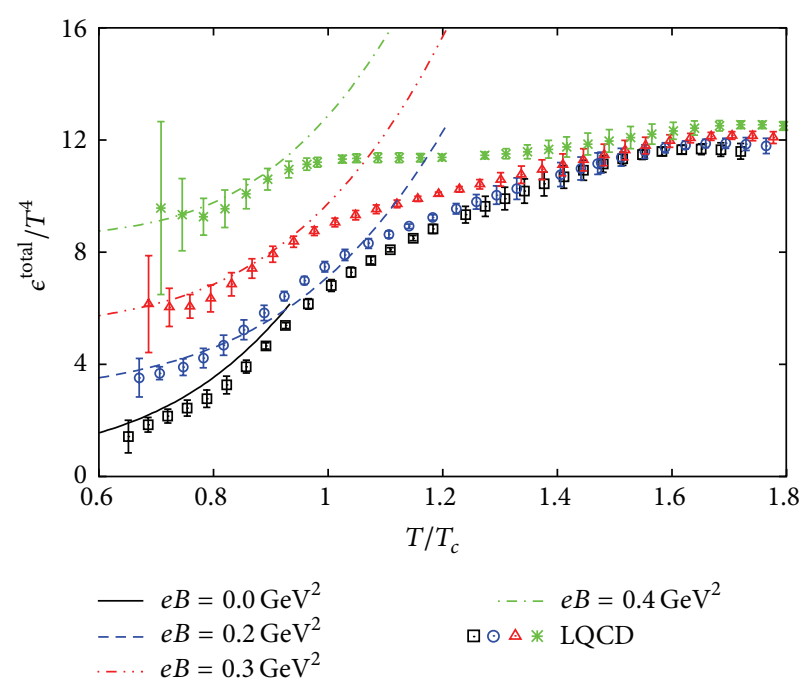

(a)

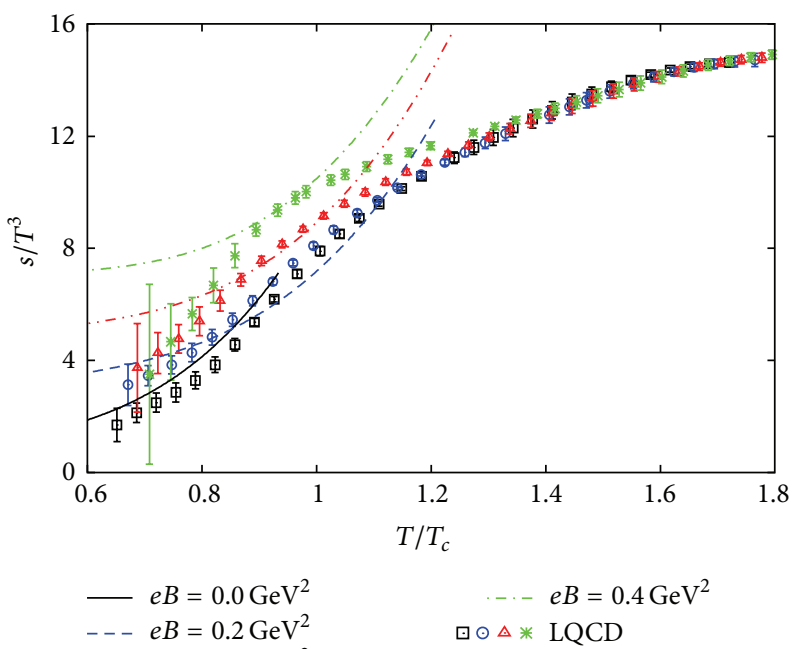

(c)

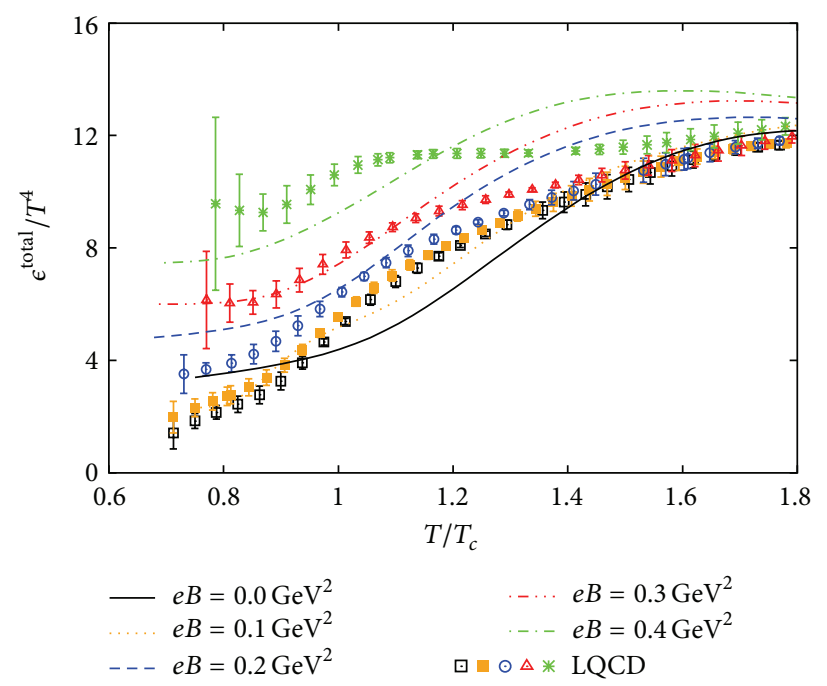

(b)

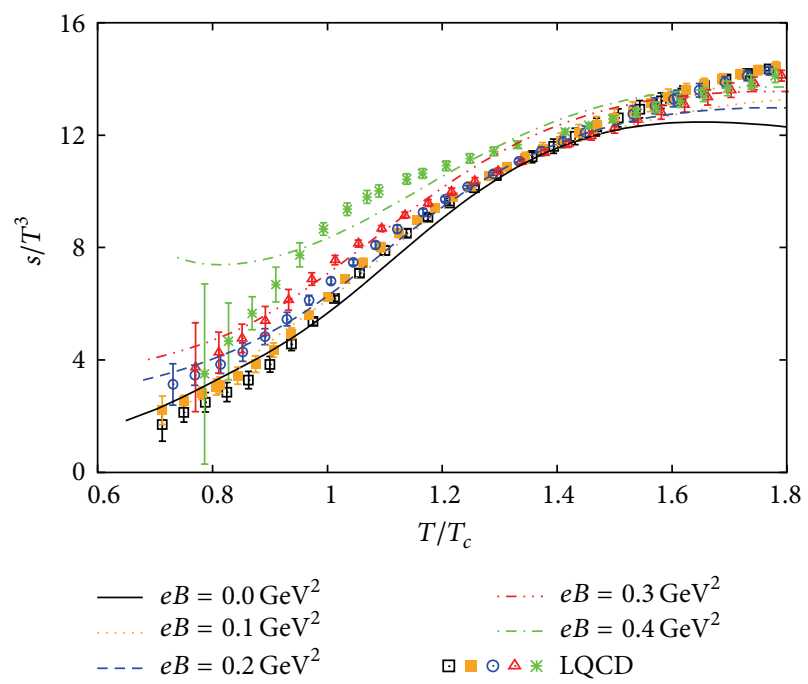

(d)

FIgURE 3: The same as in Figure 1 but for normalized total energy density (21) in (a, b) and normalized entropy density in (c, d).

In presence of finite magnetic field, both HRG and PLSM are individually compared with recent LQCD calculations at $e B=0.0-0.4 \mathrm{GeV}^{2}$. PLSM shows an excellent agreement with the lattice QCD results at vanishing and nonvanishing magnetic field. The construction of the PLSM plays an important role. The inclusion of the magnetic field can be partly modelled by changing both dispersion relations equations (2) and (3) for HRG and PLSM approaches, respectively. In this doing, the momentum phase-space should be reduced as given in (6) and (18) and also scaled to the quark and hadron electric charges and the magnetic field. This latter process is known as dimension reduction or magnetic catalysis effect. In inserting the magnetic field to both lattice and PLSM, the procedure implemented is almost the same. The agreement between HRG and lattice is fairly good, especially below the critical temperature. The modified dispersion relation follows Lorentz invariance principle, where an extra term (due to magnetic energy) is added to the total energy. Accordingly, the entropy should be modified. The sign of the magnetization depends on the magnetic property of the QCD matter. From both models and lattice calculations, we conclude that the QCD matter is likely paramagnetic. The temperature dependence is monotonic. The magnetization from HRG fairly qualitatively agrees with the lattice magnetization, while PLSM excellently reproduces it (quantitatively agrees). The phase transition at critical temperature seems not affecting the paramagnetic property.

Furthermore, we conclude that raising the magnetic field strength increases the thermodynamic quantities, especially in the hadronic phase. At high temperatures (partonic phase), the thermodynamics is apparently limited to the StefanBoltzmann limits. The latter are likely slightly affected by the magnetic field strength. At high temperatures, PLSM overestimated lattice magnetization and energy density. This might be explained from the limitation of PLSM at such high temperatures. The latter can be fine-tuned while estimating chiral 
condensates, $\sigma_{l}$ and $\sigma_{s}$, and deconfinement order parameters, $\phi$ and $\phi^{*}$, respectively. The possible thermal-modifications of various thermodynamic quantities calculated from both HRG and PLSM approaches lead to the conclusion that increasing the magnetic field strength reduces the critical temperature, that is, inverse magnetic catalysis.

\section{Conflict of Interests}

The authors declare that there is no conflict of interests regarding the publication of this paper.

\section{References}

[1] V. V. Skokov, A. Y. Illarionov, and V. D. Toneev, "Estimate of the magnetic field strength in heavy-ion collisions," International Journal of Modern Physics A, vol. 24, no. 31, pp. 5925-5932, 2009.

[2] W. Deng and X. Huang, "Event-by-event generation of electromagnetic fields in heavy-ion collisions," Physical Review C, vol. 85, Article ID 044907, 2012.

[3] J. O. Andersen and R. Khan, "Chiral transition in a magnetic field and at finite baryon density," Physical Review D, vol. 85, no. 6, Article ID 065026, 2012.

[4] A. Lazarian, S. Boldyrev, C. Forest, J. Sarff, and P. Terry, "Understanding of the role of magneticfields: galactic perspective," http://arxiv.org/abs/0902.3618.

[5] G. Endrodi, "QCD equation of state at nonzero magnetic fields in the Hadron Resonance Gas model," Journal of High Energy Physics, vol. 2013, no. 23, 2013.

[6] A. Bhattacharyya, S. K. Ghosh, R. Ray, and S. Samanta, "Exploring effects ofmagnetic field on the Hadron Resonance Gas," http://arxiv.org/abs/1504.04533.

[7] G. S. Bali, F. Bruckmann, G. Endrödi et al., "The QCD phase diagram for external magnetic fields," Journal of High Energy Physics, vol. 2012, no. 2, article 044, 2012.

[8] G. S. Bali, F. Bruckmann, G. Endrodi, F. Gruber, and A. Schafer, "Magnetic field-induced gluonic (inverse) catalysis and pressure (an)isotropy in QCD," Journal of High Energy Physics, vol. 2013, no. 4, article 130, 2013.

[9] G. S. Bali, F. Bruckmann, G. Endrődi, and A. Schäfer, "Magnetization and pressures at nonzero magnetic fields in QCD," in Proceedings of the 31st International Symposium on Lattice Field Theory (LATTICE '13), Mainz, Germany, July-August 2013.

[10] L. Levkova and C. DeTar, "Quark-Gluon plasma in an external magnetic field," Physical Review Letters, vol. 112, no. 1, Article ID 012002, 2014.

[11] C. Bonati, M. D'Elia, M. Mariti, M. Mesiti, F. Negro, and F. Sanfilippo, "Anisotropy of the quark-antiquark potential in a magnetic field," Physical Review D, vol. 89, Article ID 114502, 2014.

[12] G. S. Bali, F. Bruckmann, G. Endrődi, S. D. Katz, and A. Schäfer, "The QCD equation of state in background magnetic fields," Journal of High Energy Physics, vol. 2014, article 177, 2014.

[13] S. P. Klevansky, "The Nambu-Jona-Lasinio model of quantum chromodynamics," Reviews of Modern Physics, vol. 64, no. 3, pp. 649-708, 1992.

[14] P. G. Allen and N. N. Scoccola, "Quark matter under strong magnetic fields in SU(2) NJL-type models: parameter dependence of the cold dense matter phase diagram," Physical Review D, vol. 88, no. 9, Article ID 094005, 2013.
[15] D. P. Menezes, M. B. Pinto, S. S. Avancini, A. P. Martínez, and C. Providência, "Quark matter under strong magnetic fields in the Nambu-Jona-Lasinio model," Physical Review C, vol. 79, no. 3, Article ID 035807, 2009.

[16] K. Fukushima, M. Ruggieri, and R. Gatto, "Chiral magnetic effect in the Polyakov-Nambu-Jona-Lasinio model," Physical Review D, vol. 81, no. 11, Article ID 114031, 2010.

[17] A. N. Tawfik and N. Magdy, "SU(3) Polyakov linear- $\sigma$ model in an external magnetic field," Physical Review C, vol. 90, no. 1, Article ID 015204, 2014.

[18] A. N. Tawfik and N. Magdy, "SU(3) Polyakov linear- $\sigma$ model in magnetic fields: thermodynamics, higher-order moments, chiral phase structure, and meson masses," Physical Review C, vol. 91, no. 1, Article ID 015206, 2015.

[19] R. Gatto and M. Ruggieri, "Quark matter in a strong magnetic background," in Strongly Interacting Matter in Magnetic Fields, vol. 871 of Lecture Notes in Physics, pp. 87-119, Springer, Berlin, Germany, 2013.

[20] D. E. Kharzeev, K. Landsteiner, A. Schmitt, and H.-U. Yee, "Strongly interacting matter in magnetic fields: a guide to this volume," in Strongly Interacting Matter in Magnetic Fields, vol. 871 of Lecture Notes in Physics, pp. 1-11, Springer, Berlin, Germany, 2013.

[21] J. O. Andersen and W. R. Naylor, "Phase diagram of QCD in a magnetic field: a review," http://arxiv.org/abs/1411.7176.

[22] I. A. Shovkovy, "Magnetic catalysis: a review," Lecture Notes in Physics, vol. 871, pp. 13-49, 2013.

[23] L. Landau and E. Lifshits, The Classical Theory of Fields: Course on Theoretical Physics, Pergamon Press, Oxford, UK, 1971.

[24] L. Landau, E. Lifshitz, and L. Pitaevskii, Electrodynamics of Continuous Media, Course of Theoretical Physics, ButterworthHeinemann, 1995.

[25] E. S. Fraga and A. J. Mizher, "Chiral transition in a strong magnetic background," Physical Review D, vol. 78, no. 2, Article ID 025016, 2008.

[26] F. Karsch, K. Redlich, and A. Tawfik, "Hadron resonance mass spectrum and lattice QCD thermodynamics," European Physical Journal C, vol. 29, no. 4, pp. 549-556, 2003.

[27] F. Karsch, K. Redlich, and A. Tawfik, "Thermodynamics at non-zero baryon number density: a comparison of lattice and hadron resonance gas model calculations," Physics Letters B, vol. 571, no. 1-2, pp. 67-74, 2003.

[28] K. Redlich, F. Karsch, and A. Tawfik, "Heavy-ion collisions and lattice QCD at finite baryon density," Journal of Physics G: Nuclear and Particle Physics, vol. 30, no. 8, pp. S1271-S1274, 2004.

[29] A. Tawfik, "QCD phase diagram: a comparison of lattice and hadron resonance gas model calculations," Physical Review D, vol. 71, no. 5, Article ID 054502, 2005.

[30] A. Tawfik, "Influence of strange quarks on the QCD phase diagram and chemical freeze-out," Journal of Physics G, vol. 31, no. 6, Article ID S1105, 2005.

[31] A. N. Tawfik, "Equilibrium statistical-thermal models in highenergy physics," International Journal of Modern Physics A, vol. 29, no. 17, Article ID 1430021, 2014.

[32] R. Venugopalan and M. Prakash, "Thermal properties of interacting hadrons," Nuclear Physics A, vol. 546, no. 4, pp. 718-760, 1992.

[33] A. Tawfik, "A universal description for the freezeout parameters in heavy-ion collisions," Nuclear Physics A, vol. 764, pp. 387-392, 2006. 
[34] A. Tawfik, "Condition driving chemical freeze-out," Europhysics Letters, vol. 75, no. 3, pp. 420-426, 2006.

[35] H. Taya, "Hadron masses in strong magnetic fields," Physical Review D, vol. 92, no. 1, Article ID 014038, 2015.

[36] E. V. Luschevskaya and O. V. Larina, "The $\rho$ and $A$ mesons in strong abelian magnetic field in $S U(2)$ lattice gauge theory," Nuclear Physics B, vol. 884, pp. 1-16, 2014.

[37] I. A. Shovkovy, "Magnetic catalysis: a review," in Strongly Interacting Matter in Magnetic Fields, vol. 871 of Lecture Notes in Physics, pp. 13-49, Springer, Berlin, Germany, 2013.

[38] O. Scavenius, Á. Mócsy, I. N. Mishustin, and D. H. Rischke, "Chiral phase transition within effective models with constituent quarks," Physical Review C, vol. 64, no. 4, Article ID 045202, 2001.

[39] M. Gell-Mann and M. Levy, "The axial vector current in beta decay," Nuovo Cimento, vol. 16, article 53, 1960.

[40] S. Gasiorowicz and D. A. Geffen, "Effective Lagrangians and field algebras with chiral symmetry," Reviews of Modern Physics, vol. 41, pp. 531-573, 1969.

[41] A. Tawfik, N. Magdy, and A. Diab, "Polyakov linear SU(3) $\sigma$ model: features of higher-order moments in a dense and thermal hadronic medium," Physical Review C, vol. 89, Article ID 055210, 2014.

[42] A. Tawfik and A. Diab, "Polyakov SU(3) extended linear- $\sigma$ model: sixteen mesonic states in chiral phase structure," Physical Review C, vol. 91, no. 1, Article ID 015204, 2015.

[43] A. M. Polyakov, "Thermal properties of gauge fields and quark liberation," Physics Letters B, vol. 72, no. 4, pp. 477-480, 1978.

[44] C. Ratti, M. A. Thaler, and W. Weise, "Phases of QCD: lattice thermodynamics and a field theoretical model," Physical Review D, vol. 73, no. 1, Article ID 014019, 2006.

[45] B. Schaefer, J. M. Pawlowski, and J. Wambach, "Phase structure of the Polyakov-quark-meson model," Physical Review D, vol. 76, no. 7, Article ID 074023, 2007.

[46] S. Rößner, C. Ratti, and W. Weise, "Polyakov loop, diquarks, and the two-flavor phase diagram," Physical Review D, vol. 75, no. 3, Article ID 034007, 2007.

[47] K. Fukushima, "Phase diagrams in the three-flavor NambuJona-Lasinio model with the Polyakov loop," Physical Review D, vol. 77, no. 11, Article ID 114028, 2008.

[48] G. Endrodi, "Critical point in the QCD phase diagram for extremely strong background magnetic fields," Journal of High Energy Physics, vol. 2015, article 173, 2015. 

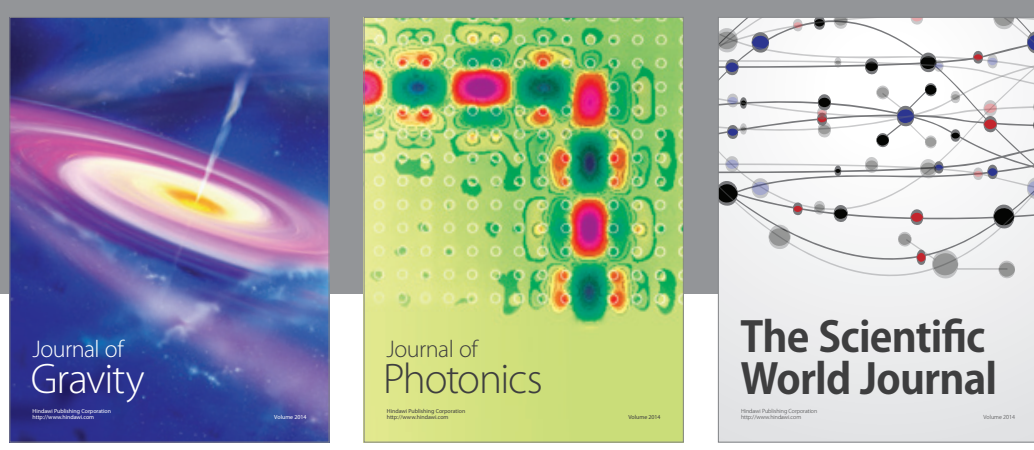

The Scientific World Journal
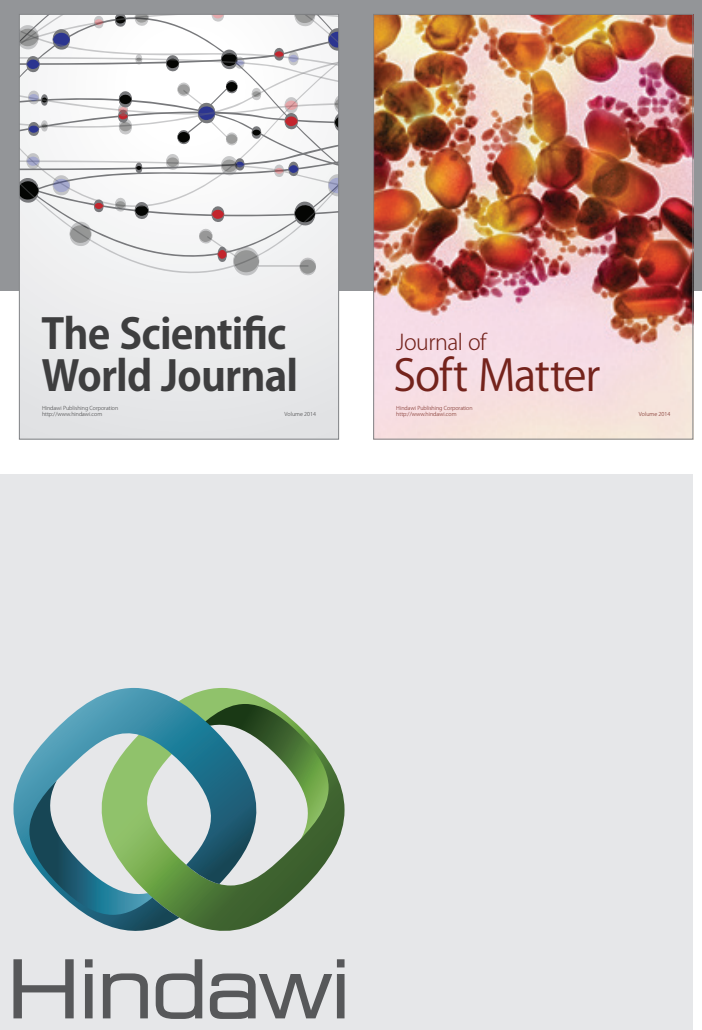

Submit your manuscripts at

http://www.hindawi.com

nternational Journal of

Statistical Mechanics
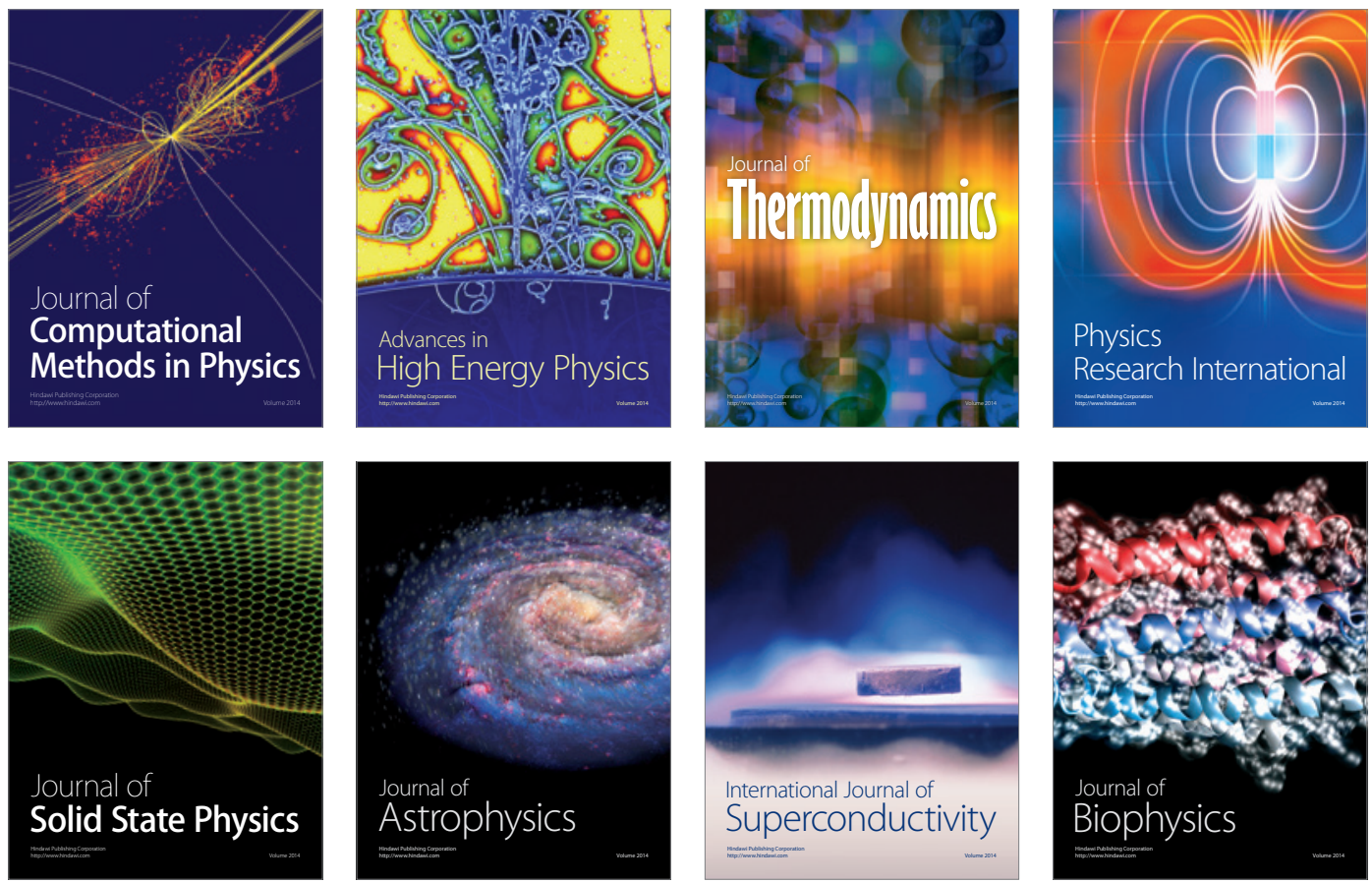
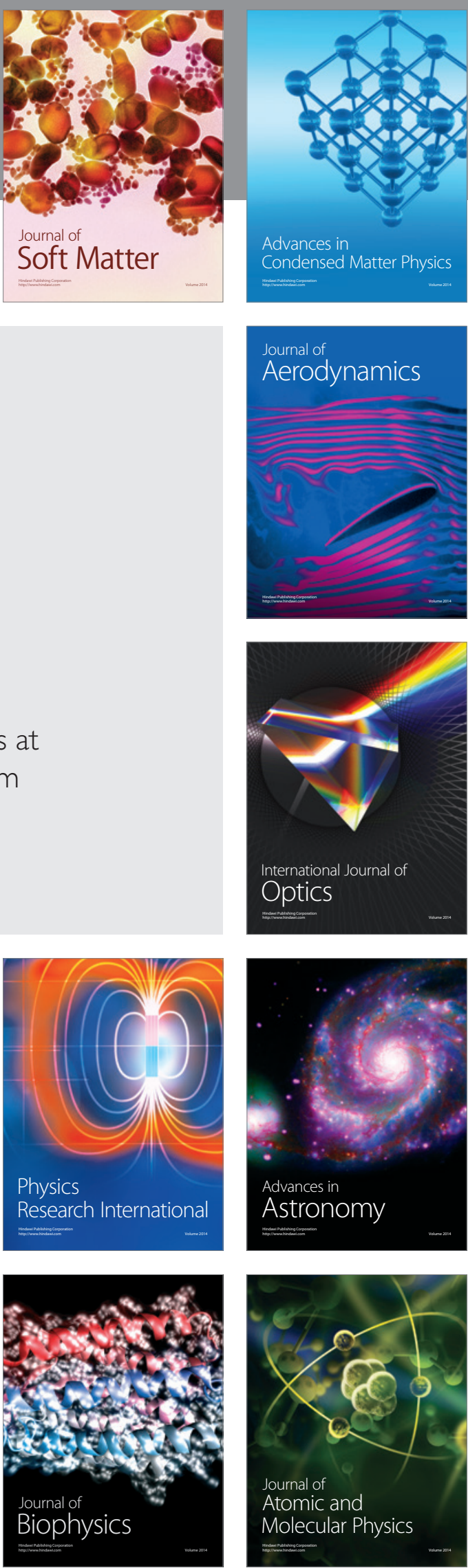